Table 5 Rank Order Correlations between the Change in the Excretion of Urinary $p$-Tyramine and Some EEG Measures

\begin{tabular}{lccc} 
& $\begin{array}{c}\text { Post-surgical } \\
(n=11)\end{array}$ & $\begin{array}{c}\text { Non-surgical } \\
(n=12)\end{array}$ & $\begin{array}{c}\text { All patients } \\
(n=23)\end{array}$ \\
MIA decrease & 0.13 & -0.19 & -0.08 \\
CV increase & $0.67 *$ & 0.41 & $0.39 *$ \\
\hline
\end{tabular}

* Significant at the $95 \%$ confidence level.

alpha frequency content. From integrated EEG measures it was apparent that before the drug treatment signal variability (CV scores) extended down to extremely low values, even lower in fact than those seen in chronic withdrawn schizophrenics or hallucinating acute schizophrenics. The MIA tended to be higher and the CV significantly lower than normal values. The changes occurring during L-dopa therapy were different depending on whether the patient had previously undergone brain surgery. In the non-surgical cases $(n=27)$ the $\mathrm{CV}$ scores increased, while in all cases $(n=40)$ the MIA scores decreased. Significant correlations were observed between increases in CV and decreases on the Columbia Scale of Disability ${ }^{17}$; changes in the tremor ratings correlated significantly with decreases in MIA and increases in CV.

In patients where both EEG and amines were analysed $(n=23)$ a significant correlation (Table 5) existed between increases in CV and decreases in urinary p-tyramine excretion. In post-surgical cases this correlation was even more marked. This is not the first time that a correlation between the EEG and urinary unconjugated $p$-tyramine has been observed. In a heterogenous population of schizophrenic patients a significant correlation existed between those patients exhibiting abnormalities in their EEG and above normal levels of urinary p-tyramine ${ }^{8}$.

It is a puzzle that urinary free $p$-tyramine should decrease during L-dopa treatment while tryptamine remains relatively unchanged. If the amines were formed simply by decarboxylation of their parent amino-acids recently available kinetic data on the responsible enzyme-L-aromatic amino-acid decarboxylase-indicate that tryptophan would be much more easily decarboxylated ${ }^{18}$ than $p$-tyrosine. If the newly discovered enzyme, dopamine dehydroxylase ${ }^{19}$, were involved it might be expected that $p$-tyramine excretion would increase on account of the high levels of circulatory L-dopa and hence presumably dopamine. It is quite possible that dopa, dopamine or one of the recently postulated products of dopa metabolism ${ }^{20}$ might act as an inhibitor of one or both of the above mentioned enzymes as is the case, for example, between dopa and tyrosine aminotransferase ${ }^{21}$.

The recent identification of $p$-tyramine and other noncatecholic amines (octopamine, phenylethylamine and tryptamine) in rat t2-24 and human brain $^{25}$, and the demonstration that $p$-tyramine is present in significant quantities and distributed similarly to noradrenaline in both the rat and $\operatorname{man}^{25}$, suggest that this particular amine (and probably the others) possesses some neurophysiological role. The observed low levels, in Parkinson patients, of dopamine in the urine and basal ganglia and the increased urinary level of $p$-tyramine together with the recent identification of cerebral dehydroxylation of dopamine to $p$-tyramine and the results contained in this paper seem to suggest an involvement of $p$-tyramine in the aetiology of Parkinsonism at least in a proportion of cases. Recent mass spectrometric analyses have shown that rat brain tyramine is almost, if not entirely, the para isomer ${ }^{26}$. This means that it is the meta hydroxyl group that is lost from dopamine during dehydroxylation so that little, if any, meta tyramine can be present. Although this latter observation is so far confined to the rat, it is important in view of the recent suggestion by Sandler et al. ${ }^{27,28}$ that perhaps in Parkinsonism intestinal para dehydroxylation of dopa occurs with the resultant meta tyrosine being transported to the brain where it is converted to meta tyramine; this then acts to protect dopamine.
We thank the Psychiatric Services Branch, Province of Saskatchewan, the Medical Research Council of Canada and Hoffmann-LaRoche (Canada) for financial support; Dr A. H. Rajput for urine samples from his patients, and Mrs Donna Wilson, Miss Joan Read, Mrs Kathy Bergstrom, Mr R. Majer and $\mathrm{Mr}$ W. Hrychuk for technical assistance.

Alan A. Boulton

GORDON L. MARJERRISON

Psychiatric Research Unit and

Department of Psychiatry,

University Hospital,

Saskatoon, Saskatchewan

Received June 17; revised August 10, 1971.

${ }^{1}$ Kakimoto, Y., and Armstrong, M. D., J. Biol. Chem., 237, 208 (1962).

${ }^{2}$ Boulton, A. A., Pollitt, R. J., and Majer, J. R., Nature, 215, 132 (1967).

3 Smith, I., and Kellow, A. H., Nature, 221, 1261 (1969).

4 Boulton, A. A., Prog. Neurogenetics, 1, 437 (1967).

5 Asatoor, A. M., Clin. Chim. Acta, 22, 223 (1968).

6 DeQuattro, V. L., and Sjoerdsma, A., Clin. Chim. Acta, 16, 227 (1967).

${ }^{7}$ Perry, T. L., Hestrin, M., MacDougall, L., and Hansen, S., Clin. Chim. Acta, 14, $116(1966)$.

8 Boulton, A. A., Marjerrison, G. L., and Majer, J. R., Symp. Clin. Biol. Res. Schizophrenia, Leningrad (September 1970, in the press).

9 Boulton, A. A., Meth. Biochem. Anal., 16, 327 (1968).

10 Marjerrison, G. L., Boulton, A. A., and Rajput, A., Intern. J. Clin. Pharmacol., 4, 263 (1971).

11 Boulton, A. A., in L-Dopa and Parkinsonism (edit. by Barbeau, A., and McDowell, F. H.) (F. A. Davis, Philadelphia, 1970).

12 Barbeau, A., Murphy, G. F., and Sourkes, T. L., Science, 133, 1706 (1961).

13 Horneykiewicz, O., Pharmacol. Rev., 18, 925 (1966).

14 Cotzias, G. C., in L-Dopa and Parkinsonism (edit. by Barbeau, A., and McDowell, F. H.) (F. A. Davis, Philadelphia, 1970).

15 Goldstein, L., Sugerman, A. A., Stolberg, H., Murphree, H. B., and Pfeiffer, C. C., EEG Clin. Neurophysiol., 19, 350 (1965).

16 Marjerrison, G. L., Krause, A. E., and Keogh, R. P., EEG Clin. Neurophysiol., 24, 35 (1968).

17 Yahr, M. D., Duvoisin, R. C., Schear, M. J., Barrett, R. E., and Hoehn, M. M., Arch. Neurol., 21, 343 (1969)

18 Christenson, J. G., Pairman, W., and Udenfriend, S., Arch. Biochem. Biophys., 141, 356 (1970).

19 Boulton, A. A., and Quan, Lillian, Canad. J. Biochem., 48, 1287 (1970).

${ }^{20}$ Sourkes, T. L., Nature, 229, 413 (1971)

21 Fellman, J. H., and Roth, E. S., Biochemistry, 10, 408 (1971).

22 Boulton, A. A., and Majer, J. R., Nature, 225, 658 (1970).

${ }_{23}$ Majer, J. R., and Boulton, A. A., J. Chromatog., 48, 322 (1970).

24 Boulton, A. A., and Majer, J. R., Methods in Neurochemistry, 1 (edit. by Rodnight, R., and Marks, N.) (Plenum, New York, in the press).

25 Majer, J. R., and Boulton, A. A., Meth. Biochem. Anal., 49, 993 (1971).

26 Boulton, A. A., and Majer, J. R., Canad. J. Biochem. (in the press).

${ }^{27}$ Sandler, M., Karoum, F., Ruthven, C. R. J., and Calne, D. B., Science, 166, 1417 (1969).

${ }^{28}$ Sandler, M., Goodwin, B. L., Ruthven, C. R. J., and Calne, D. B., Nature, 229, 414 (1971).

29 Udenfriend, S., in Fluorescence Assay in Biology and Medicine, 164 (Academic Press, New York, 1962).

\section{Australia Antigen as a Marker of Propagation of the Serum Hepatitis Virus in Liver Cultures}

ATTEMPTS to isolate the human hepatitis viruses in tissue culture have resulted in a collection of "hepatitis-candidate" viruses, none of which has since been shown to be the causal agent of human hepatitis ${ }^{1}$. The discovery of Australia antigen provided a specific serological marker of infection with or carriage of the serum hepatitis virus, although the nature of the antigen remains unsettled ${ }^{2}$.

Preliminary observations on the irregular production of cytopathic changes by purified fractions of serum containing 


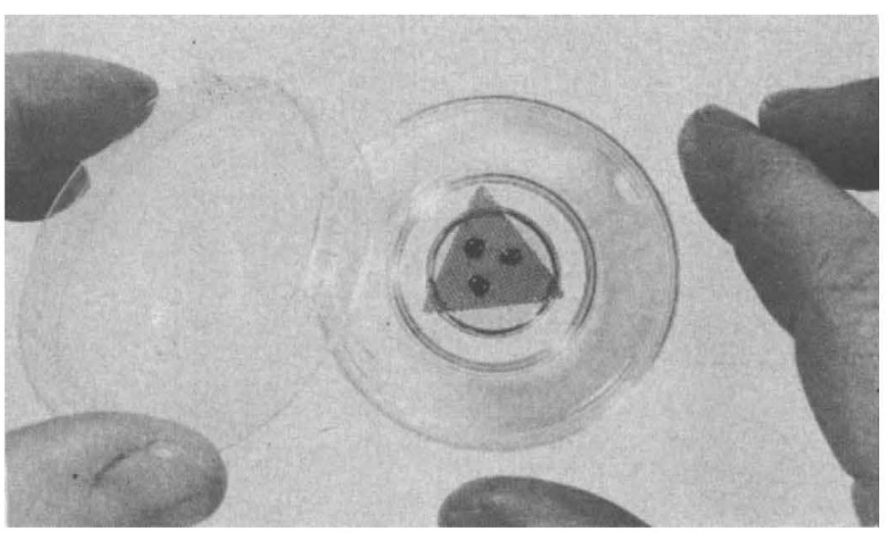

Fig. 1 Organ culture of human embryo liver $24 \mathrm{~h}$ after preparation.

Australia antigen in monolayer cultures of differentiated human embryo liver cells ${ }^{3}$ proved unpredictable. More recently, however, the induction of progressive changes in such cultures has been reported after inoculation with a known infective hepatitis serum. There was progressive involvement of the normal cellular components of the cultured liver cells, as demonstrated by specific fluorescent antibody attachment, first in the cytoplasm, then in the perinuclear membrane and finally condensation in the nucleoli. Similar progressive changes have been observed in cells of liver cultures inoculated with supernatant fluid which has been passaged in culture once ${ }^{4}$. Virtually identical fluorescent changes have been reported in liver cells cultured from biopsy material obtained from a patient with circulating Australia antigen. After six passages of the cells, the cultured cells still contained Australia antigen in the nuclei and the antigen was also detected by radioimmunoassay in the supernatant fluid ${ }^{5}$.

More reliable changes can be obtained, however, by using small fragments of tissue maintained in synthetic media as organ cultures. These can be used to propagate certain viruses that are difficult to grow in conventional cell cultures. A technique has now been developed for maintaining human embryo liver organ cultures for at least 10 days. These cultures support the growth of type 3 adenovirus $^{6}$, and they have been used for the inoculation of serum specimens from patients and carriers of type B viral hepatitis with some encouraging results $^{7}$.

Human livers, obtained after abdominal hysterotomy, from 10-16-week-old embryos were prepared for organ culture as soon as possible after dissection, usually within 3-4 h. The tissue was washed in Hanks balanced salt solution and cut into fragments of approximately $2 \mathrm{~cm}^{3}$. Three fragments were placed on sterilized stainless steel grids, which fit into the centre well of a Falcon disposable plastic organ culture dish (Fig. 1). Eagle's minimum essential medium with $0.2 \%$ bovine serum albumin, $100 \mathrm{U}$ of penicillin, $100 \mu \mathrm{g}$ of streptomycin and $50 \mathrm{U}$ of 'Nystatin' $\mathrm{ml}^{-1}$, was added to the centre well until the tissue was bathed but not immersed in the medium $(0.8 \mathrm{ml}$.). The $p \mathrm{H}$ of the medium was maintained at 6.8 and the cultures incubated at $35^{\circ} \mathrm{C}$. A sample of $0.1 \mathrm{ml}$. of serum from three different sources was used for inoculation. Serum P.J.G. was obtained from a former blood donor who was implicated in three cases of serum hepatitis. His serum was shown to be infective by volunteer studies and Australia antigen has been demonstrated repeatedly in his serum $^{8}$. Serum R.B. was collected from a jaundiced patient admitted to a London hospital with viral hepatitis and Australia antigen was subsequently detected serologically. Serum G.C. was obtained from an apparently healthy volunteer blood donor whose blood had caused two deaths from post-transfusion hepatitis in transfused recipients. Australia antigen was demonstrated in this serum by routine serological tests and by electron microscopy.

The inoculated sera were incubated with the organ cultures for $72 \mathrm{~h}$ at $35^{\circ} \mathrm{C}$. The culture fluid was removed with a fine pipette, replaced with fresh medium and the incubation continued. The culture fluid together with the liver fragments were collected after 6 and 8 days. The material was then rapidly frozen and thawed three times and the resulting fluid used for examination for Australia antigen. The antigen could not be demonstrated in the supernatant fluid by immunodiffusion, complement fixation or radioimmunoassay after 3 days of incubation. Progressive rise in the titre of Australia antigen was obtained with serum G.C. and serum R.B., from a complement fixing titre of $1: 4$ at 6 days to $1: 128$ after
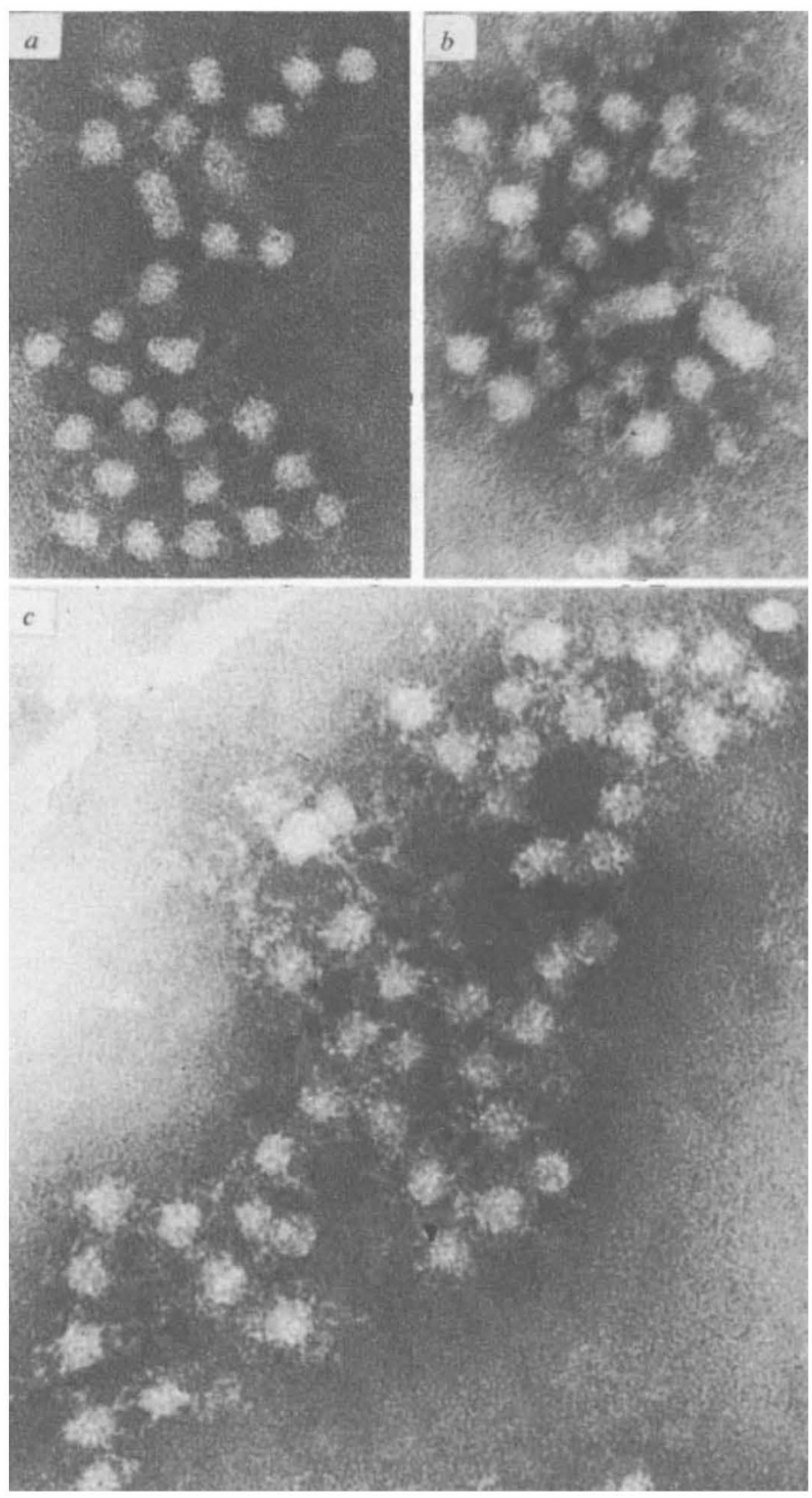

Fig. $2 a$, A small aggregate of Australia antigen particles present in the serum of R.B. This patient had clinical serum hepatitis and the serum was one of the three sera used for inoculation of the organ cultures $(\times 300,000)$. $b$, This micrograph was obtained from the organ culture fluid 6 days after inoculation with the serum shown in Fig. $2 a$. Only very occasional small aggregates of the type shown here were found $(\times 300,000)$. $c$, After 8 days growth in organ culture, examination of the culture fluid in the electron microscope revealed numerous aggregates showing the typical morphology of Australia antigen. The aggregate illustrated here has been chosen because it is possible to see bridging of the particles by antibody present in a serum containing specific anti-Au globulin $(\times 300,000)$. 
Fig. 3 Using as inoculum the material harvested from the experiment illustrated in Fig. $2 c$, an additional pass in organ culture was carried out At day 8 of this first passage the electron microscope revealed the presence of enormous numbers of particles having the typical morphology of Australia antigen and aggregated by antibody specific to it. The micrograph shows only the thin edge of an aggregate which was itself $3 \mu \mathrm{m}$ in diameter $(\times 80,000)$

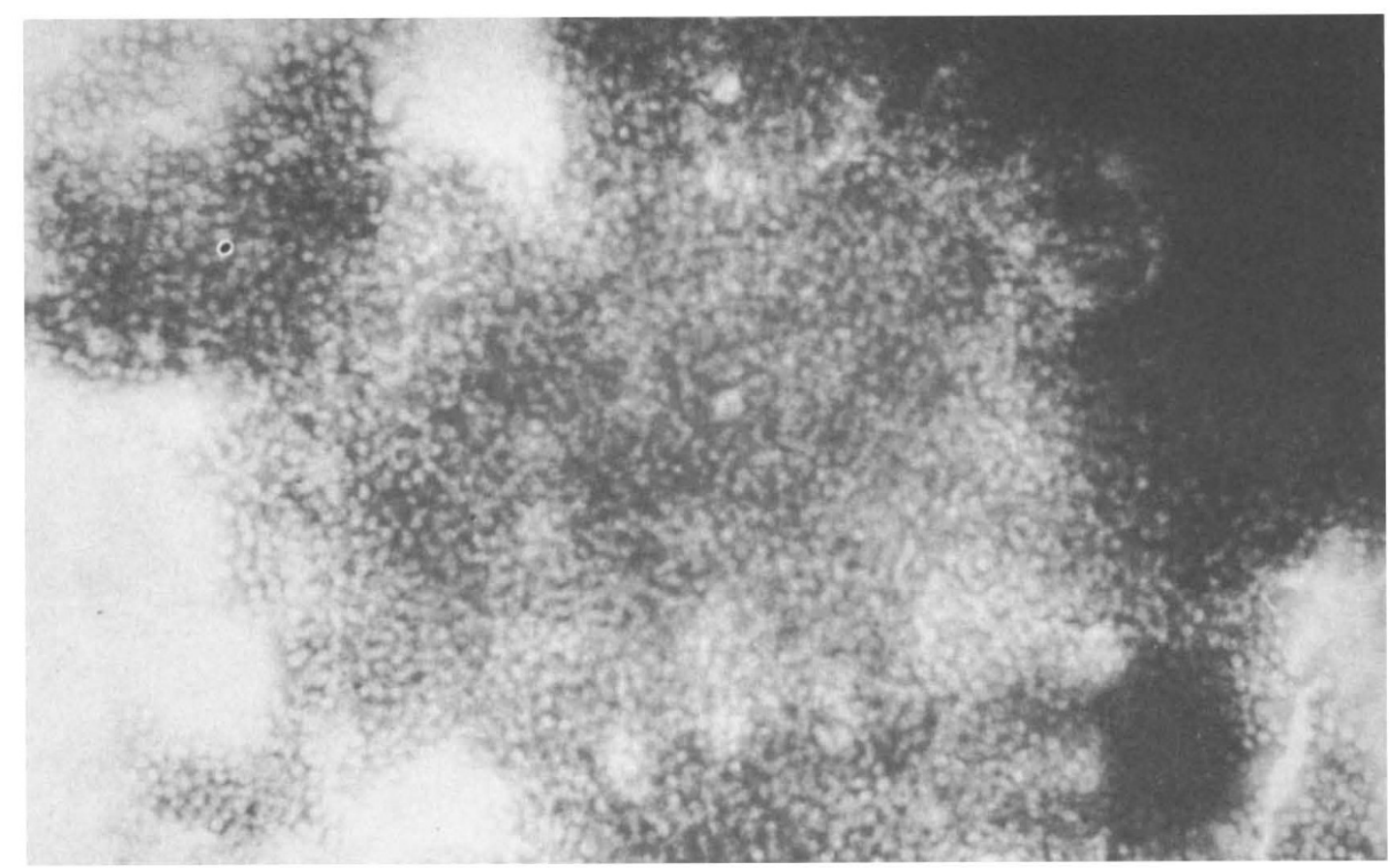

8 days in culture. In the two dimensional micro-Ouchterlony technique there was an increase in precipitin titre from 0 at 6 days to $1: 4$ on day 8 .

The specimens for electron microscopy were fluids from organ cultures containing considerable amounts of cell debris, so we looked for aggregates of antigen rather than individual particles 9 . This was achieved by adding $0.1 \mathrm{ml}$. of a $1: 5$ dilution of haemophilic serum, known to contain specific antibody to the Australia antigen, to $0.5 \mathrm{ml}$. of the organ culture fluid. The tubes were then incubated for $30 \mathrm{~min}$ at $37^{\circ} \mathrm{C}$ and centrifuged for $1 \mathrm{~h}$ at 18,000 r.p.m. in a Sorvall $\mathrm{RC} 2 \mathrm{~b}$ centrifuge. The supernatant was discarded and the pellets used for negative staining as follows. Immediately before examination the pellet was resuspended in approximately $0.1 \mathrm{ml}$. of distilled water and an aliquot of this mixed with an equal quantity of $3 \%$ phosphotungstic acid adjusted to $p \mathrm{H} 6.0$. A drop of this mixture was then placed on a 400 mesh carbonformvar coated grid and examined in a Philips EM 300, chiefly at a plate magnification of $\times 60,000$.

In each of the three sera used were found the particles now associated with Australia antigen. In each case the addition of antiserum had clumped the particles and direct bridging by antibody molecules could be seen (Fig. 2c). Tissue culture material from control specimens contained numerous small particles in the size range of the $200 \AA$ diameter Australia antigen particles. These particles, however, were never clumped by antibody and on closer examination appeared to be the normal constituents of any tissue culture preparation. On the sixth day of the original cultures, which were infected with G.C. and R.B., the electron microscope revealed that a small number of aggregates built up with typical Australia antigen particles were present. These immune aggregates were small and could be found only by diligent searching (Fig. 2b). By day 8 of this series, the grids examined contained numerous large aggregates again typical of Australia antigen particles. The aggregate shown in Fig. $2 c$ has been chosen for illustration because it is possible to see the antibody linkage by specific anti-Au serum.

The control preparations and cultures inoculated with a normal serum remained negative by all serological tests and on electron microscopic examination.

The fluid collected from the 8 day cultures inoculated with G.C. and R.B. was passaged into fresh organ cultures. There was no detectable complement fixing activity in the tissue fluid collected after 6 days and only a slight rise in the comple- ment fixing titre to $1: 2$ with the G.C. passage material at 8 days. The immunodiffusion test both on the sixth and eighth day was negative. No complement fixing nor precipitin reactions were detected in the sixth day collection from R.B. passage material. On the other hand, although complement was fixed to a titre of only $1: 4$ at 8 days with R.B. passage material, a sharply defined precipitin line was obtained in the gel diffusion test, showing a line of immunological identity with known reference Australia antigen-containing sera.

The day 6 passage material derived from G.C. showed very scanty aggregates in the electron microscope, and in the specimen from day 8 there was only a very small increase in the number of Australia antigen particles. Similarly, day 6 of the first passage material derived from R.B. revealed only small scattered aggregates of Australia antigen; but on day 8 the specimen was found to contain considerably more Australia antigen than had been seen in any of the previous specimens including the original starting material. Many of the aggregates were too large for high resolution microscopy and the aggregate shown in Fig. 3 is a thinly spread area at the edge of an aggregate, which was approximately $3 \mu$ in diameter.

It is interesting that although occasional $420 \AA$ (Dane) particles ${ }^{10}$ could be found in the original inocula these were not seen in any of the cultured specimens. On the other hand, the specimens contained approximately $200 \AA$ diameter spheres and tubules that are typical of the Australia antigen.

The passage controls and serum negative controls were entirely negative by complement fixation, immunodiffusion, electron microscopy and radioimmunoassay. The low complement fixing titres of the R.B. passage material, in the presence of marked positive precipitin reactions and numerous Australia antigen particles revealed by immune electron microscopy (Fig. 3), suggest that complement fixing activity of Australia antigen is considerably reduced after adaptation to tissue culture conditions. Further serial passages are in progress.

Finally, there was no detectable rise in the complementfixing titre nor a precipitin reaction for Australia antigen after inoculation of the third serum from P.J.G., nor on passage ${ }^{7}$.

Australia antigen is generally recognized as a specific marker of infection with serum hepatitis virus. We conclude therefore that apparent replication of the serum hepatitis virus, as far as it can be based on measurement of Australia antigen, has taken place in organ cultures of human liver. The difficulty in obtaining suitable human foetal liver and the variation 
associated with primary tissues of different sources and ages may limit the large scale use of our culture technique. The human coronaviruses, however, were initially only capable of growth in organ culture, but after a period of adaptation by this means they could be grown on standard continuous cell cultures. It may be that a period of adaptation on foetal liver organ cultures could produce the same effect for the virus of serum hepatitis.

This work is supported by grants from the World Health Organization (Virus Diseases), Pfizer Ltd, the Medical Research Council and the Department of Health and Social Security. We thank Dr H. E. M. Kay and his staff at the Royal Marsden Hospital for help with material.

\section{A. J. ZUCKERMAN}

PAMela M. Baines

\section{Hepatitis Unit,}

Department of Microbiology,

London School of Hygiene and Tropical Medicine, London WC1E 7 HT

Department of Virology, June D. Almeida

Royal Postgraduate Medical School, London $W 12$

Received December 23, 1971; revised January 4, 1972.

1 Zuckerman, A. J., Virus Diseases of the Liver, 46 (Butterworth, London, 1970).

2 Memorandum: Tests for Australia (hepatitis-associated) antigen and antibody, Bull. Wld Hlth Org., 42, 957 (1970).

3 Zuckerman, A. J., Nature, 223, 569 (1969).

4 Brighton, W. D., Taylor, P. E., and Zuckerman, A. J., Nature New Biology, 232, 57 (1971)

5 Coyne, V. E., Millman, I., and Blumberg, B. S., Bacteriol. Proc. (Abstr.), 175 (1971).

6 Baines, P. M., Taylor, P. E., and Zuckerman, A. J., Arch. Ges. Virusforsch. (in the press).

7 Zuckerman, A. J., Vox Sang., 19, 304 (1970).

8 Zuckerman, A. J., and Taylor, P. E., Nature, 223, 81 (1969).

9 Almeida, J. D., and Waterson, A. P., Adv. Virus Res., 15, 307 (1969).

10 Dane, D. S., Cameron, C. H., and Briggs, M., Lancet, i, 695 (1970).

\section{New Semi-dominant Mutation in the Syrian Hamster}

Several different mutations causing hypotrichosis are well known in both rats and mice ${ }^{1,2}$ but none have so far been described in the hamster.

In November 1968 a spontaneous semi-dominant mutation causing hypotrichosis occurred in a litter of hamsters (Mesocricetus auratus) maintained by one of us (M. K. W.). A single male animal with a "sparse coat" was noted among normal littermates. The mating was repeated twice, but no further abnormal young were observed.

The mutant male was mated to normal females of the same strain, and approximately $50 \%$ of his offspring had a sparse coat. The original male was then mated to his sparse-coated daughters, and in May 1969 the first hairless young were observed. Breeding data given in Table 1 indicate that the condition is caused by an autosomal semi-dominant mutation resembling "naked" in the mouse. Because of this resemblance,

Table 1 Breeding Results

\begin{tabular}{cccccc}
\hline \multicolumn{2}{c}{ Mating } & No. litters & \multicolumn{3}{c}{ Young weaned } \\
+ & $\delta$ & born & $+/+$ & $N /+$ & $N / N$ \\
$+1+\times+1+$ & 5 & 38 & - & - \\
$+1+\times+\mid N$ & 22 & 95 & 81 & - \\
$+\mid N \times+/ N$ & 26 & 60 & 98 & 39 \\
$+\mid N \times N / N$ & 15 & - & 61 & 42 \\
$+/+\times N / N$ & 8 & & - & 65 & - \\
$N / N \times N / N$ & 2 & & None None None \\
\hline
\end{tabular}

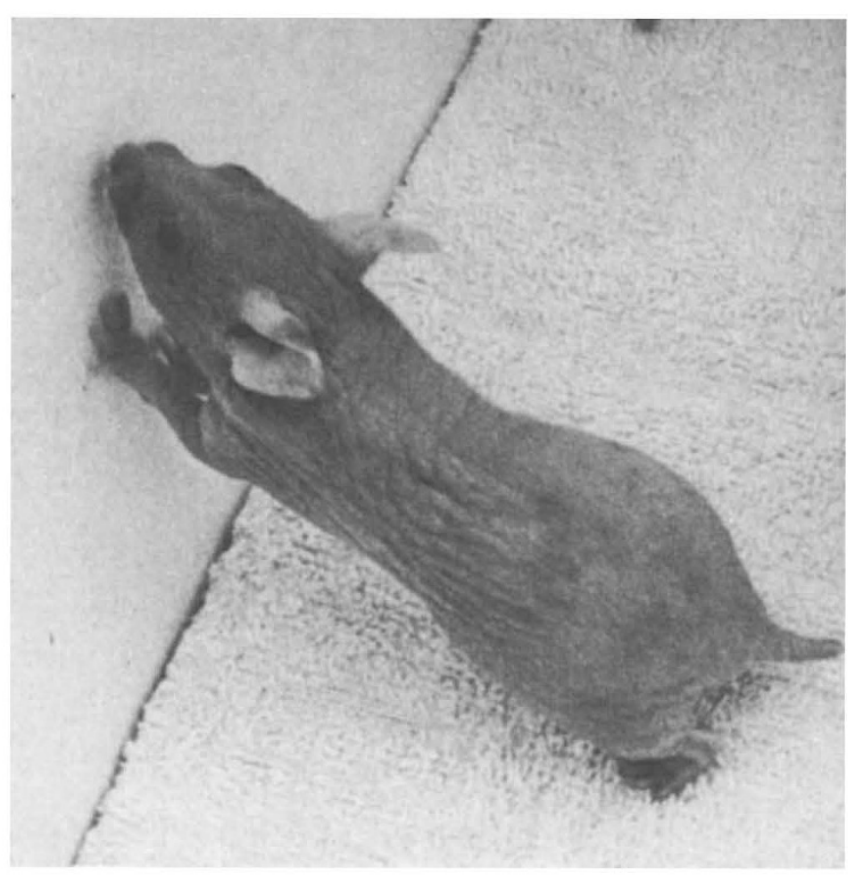

Fig. 1 The naked hamster.

the name "naked" with the gene symbol $N$ is proposed for this mutant.

Homozygous naked hamsters are completely devoid of hair apart from a very short and soft down (Fig. 1). Vibrissae are present but are soft and tend to be bent. Naked hamsters which reach weaning age are viable, though they have a reduced body weight, and are probably more sensitive to environmental factors than normal hamsters. Both male and female homozygotes are fertile though the few females that have produced young have so far been unable to rear them. The breeding data shown in Table 1 indicate that there is about a $30 \%$ deficiency of homozygotes at weaning, thus the effect on viability is much less marked than the naked mutant in the mouse ${ }^{3}$.

The onset of the condition has not been studied in detail, but naked individuals are identifiable at about 14 days of age, and there is no evidence of hair growth from then onwards. Heterozygotes are identifiable from about 14 days of age onwards by their sparse coat, which is particularly deficient on the ventral surface, though the nature of the deficiency has not yet been determined. Microscopic examination of hair clippings from heterozygotes reveals no obvious deficiency in either type or diameter of the hairs. Viability and breeding performance of the heterozygotes are normal, though there may be a slight reduction in body weight.

According to Kirkman and Algard ${ }^{4}$ the epidermis of the hamster is responsive to a variety of chemical carcinogens, producing the same types of tumours as other laboratory animals. The naked hamster may prove useful in studies of such neoplasia as it eliminates the need to shave the hamsters before applying the carcinogen.

$M R C$ Laboratory Animals Centre,

M. F. W. Festing

Woodmansterne Road,

Carshalton, Surrey

The Beeches,

M. K. WRIGHT

Latchingdon,

Chelmsford, Essex

Received September 19, 1971.

1 Robinson, R., Genetics of the Norway Rat (Pergamon Press, London, 1965).

2 Green, E. L., Biology of the Laboratory Mouse (McGraw-Hill, New York, 1966). 